\title{
The Implementation of Earth's Field NMR Spectroscopy in an Undergraduate Chemistry Laboratory
}

Patrick Bergstrom Mann, ${ }^{*}$, Samuel Clark,, Samuel T. Cahill,$\uparrow$ Craig D. Campbell,$\uparrow$ Matthew T. Harris,$\dagger$ Simon Hibble,$\uparrow$ Trang To, $\uparrow$ Andrew Worrall,$\uparrow$ Malcolm Stewart*,$\dagger$

5 †Chemistry Teaching Laboratory, Department of Chemistry, University of Oxford, South Parks Road, Oxford, OX1 3PS, United Kingdom

\section{ABSTRACT}

Earth's field nuclear magnetic resonance (EFNMR) spectroscopy offers students a unique opportunity to consolidate their understanding of NMR spectroscopic theory through hands-on practice with a simple spectrometer. A comprehensive, six-hour experiment is presented for the introduction of lowfield NMR techniques, covering spectroscopy, relaxivity, and imaging experiments in the Earth's magnetic field. This multi-faceted practical session explored the concepts of free induction decay, pulse sequences, field homogeneity, relaxation times, $J$-coupling, and magnetic resonance imaging (MRI), reinforced through a series of experiments carried out within an undergraduate teaching

15 laboratory. Students are required to alter parameters as they see fit in order to obtain data, ensuring their understanding of the theory behind NMR spectroscopy. The challenges overcome for the implementation of EFNMR spectroscopy in a modern undergraduate laboratory are also discussed and detailed instructions included for spectrometer set up.

\section{GRAPHICAL ABSTRACT}

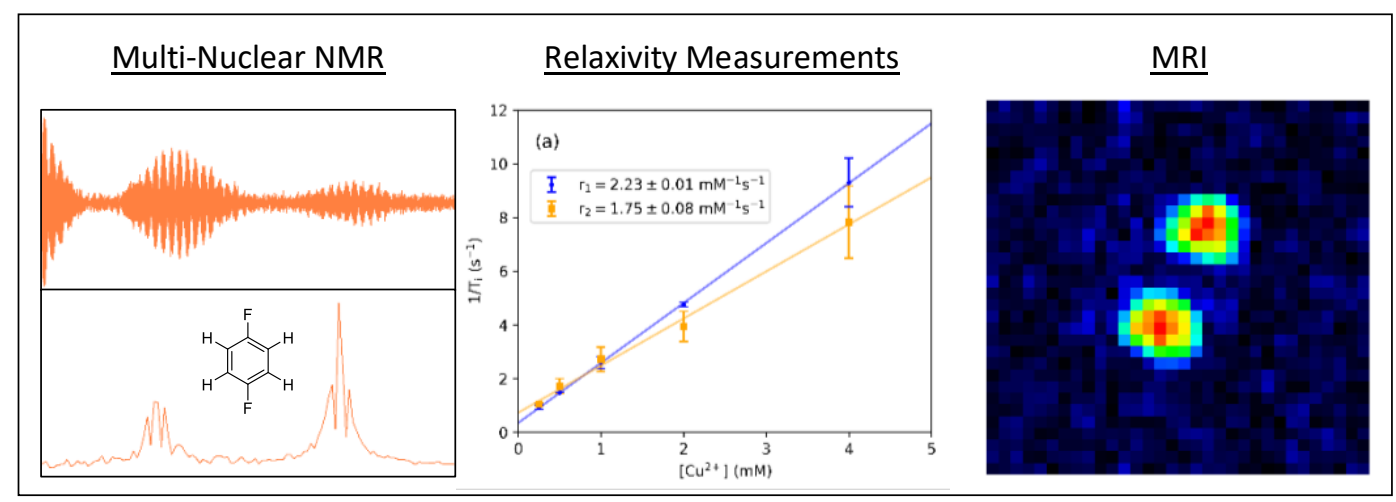

\section{KEYWORDS}

Upper-Division Undergraduate; Laboratory Instruction; Hands-On Learning/Manipulatives; NMR Spectroscopy 


\section{INTRODUCTION}

Nuclear magnetic resonance spectroscopy is a fundamental technique in many aspects of chemical research and, as such, is a key focus in undergraduate chemistry courses. However, students typically only cover NMR spectroscopic theory in lectures and workshops, focusing on data interpretation, with no practical reinforcement of concepts such as precession, pulse sequences, and relaxation times. Given the high running costs associated with high-field NMR spectrometers, it is uncommon for teaching laboratories to have dedicated instruments and, often, students submit samples without the chance to run their own spectra. This situation is changing, with advances in magnet technology that have enabled the development of benchtop NMR spectrometers with permanent magnets of 1 to 2 Tesla (T).1,2 Consequently, the accessibility of NMR spectrometers will continue to increase greatly on account of the decreases in physical spectrometer size, initial financial outlay, and running costs. Benchtop NMR spectrometers are becoming increasingly commonplace in undergraduate teaching laboratories and reports of their use in experiments is on the rise.3-7 However, whilst these experiments allow students to perform NMR spectroscopy, the instruments often remain "black boxes" that return data at the push of a button. Earth's field NMR (EFNMR) spectroscopy offers a unique opportunity for students to explore the physical aspects and fundamental concepts behind NMR spectroscopy, with a high level of control over the experiments they are running.

EFNMR spectroscopy experiments were first proposed and carried out in the 1950s but have remained a niche area of interest in chemical research.8,9 The low strength of the Earth's field (typically around $50 \mu \mathrm{T}$ ) means that chemical shift differences, which are a key component of structural elucidation by NMR spectroscopy, are not observed. The low field strength also results in a 45 small excess of nuclear spins aligned with the field, further decreasing the sensitivity of an already poorly sensitive technique.10 Coupling between heteronuclei, however, is field independent, allowing for observation and direct comparison to high-field spectra.11,12 EFNMR spectroscopy experiments are made possible by the high homogeneity of the Earth's field on a scale that allows for the use of large samples (ca. $500 \mathrm{~mL}$ ). To further improve the signal-to-noise ratio, a larger field is applied, on the order of $20 \mathrm{mT}$, which increases the net magnetization sufficiently to carry out experiments. This field is then removed prior to the NMR measurement such that the Larmor frequencies corresponding to energy level splitting in the Earth's field are observed. 
The use of EFNMR spectroscopy as an educational tool for undergraduates is where the technique excels. Students have the freedom to alter pulse sequence parameters and explore their effect on the resulting free induction decay (FID) and NMR spectra. Often in lectures and workshops, focus is placed on the interpretation of spectra and there is little connection to the actual pulse sequences that are applied to obtain the data. Despite its potential, EFNMR spectroscopy as a teaching tool has been adopted predominantly by physics departments, 13,14 with only a single report on EFNMR spectroscopy in an undergraduate chemistry setting. 15 Previously reported experiments have focused predominantly on simple water acquisitions and/or the use of NMR spectroscopy for imaging (MRI). The aspects of NMR spectroscopy that are most relevant to a chemistry undergraduate have not been discussed in detail, namely $J$-coupling and multiplicity. In our experience, the use of EFNMR probes in a busy laboratory environment is non-trivial and can be a potential barrier to implementation in an undergraduate course.

Here, an EFNMR spectroscopy experiment is outlined that covers the aspects of NMR theory most pertinent to chemical research and provide instructions on the set up of multiple probes within an inhomogeneous magnetic environment, such as a modern undergraduate laboratory. Multinuclear NMR spectroscopy, $J$-coupling, relaxation times, and weighted imaging are explored, with comparison to higher field spectra where possible. The acquisition of simple spectra gives way to imaging and the role of chemical research in the development of contrast agents for MRI. Meanwhile, the limitations of NMR spectroscopy in the Earth's field draw attention to important aspects of spectra at higher fields, such as the relation between ppm values and frequency, which are not always considered by the students.

\section{EXPERIMENTAL DETAILS}

75 Two Terranova-MRI probes (Magritek) were set up in the center of the laboratory (Figure 1), approximately three meters apart, and set up as described in the accompanying Magritek user manual. Large fluctuations in field strength within the laboratory resulted in different ${ }_{1} \mathrm{H}$ Larmor frequencies for the two probes - 2228 and $1835 \mathrm{~Hz}$, corresponding to field strengths of 52.3 and 43.1 $\mu \mathrm{T}$ respectively. However, the local fields were sufficiently homogeneous for spectroscopy and

80 imaging, with average line widths of $4 \mathrm{~Hz}$. Detailed set up instructions and a troubleshooting guide are available as part of the demonstrator notes (see Supporting Information). 
Samples were prepared in advance, with tap water, vegetable oil, $\mathrm{CuSO}_{4}(\mathrm{aq})(0.25,0.5,1$, and 2 $\mathrm{mM}), \mathrm{MnCl}_{2}(\mathrm{aq})(0.025,0.05,0.1,0.2$, and $0.3 \mathrm{mM}), \mathrm{NH}_{4} \mathrm{NO}_{3}\left(8 \mathrm{M}\right.$ in $\left.2 \mathrm{M} \mathrm{HCl}^{(\mathrm{aq})}\right)$ and 1,4-difluorobenzene contained in $500 \mathrm{~mL}$ polypropylene bottles. This minimized the chemical waste generated and the time spent preparing samples by the students.

NMReady-60 Pro (Nanalysis) $60 \mathrm{MHz}$ and Ascend (Bruker) $400 \mathrm{MHz}$ spectrometers were used to perform higher field NMR experiments for comparison to the EFNMR spectra.

The Supporting Information includes the following: a student lab script containing an introduction to NMR and EFNMR spectroscopy, suggested pre- and post-lab questions, and the experimental procedure; also included are notes for the demonstrators that cover apparatus set up, troubleshooting, model data, and answers to the pre- and post-lab questions.

\section{LEARNING OUTCOMES}

This experiment explores numerous key applications of NMR theory - spectroscopy, relaxivity and imaging. The objectives of the practical session include:

- Measurement of the Larmor frequencies of ${ }_{1} \mathrm{H}$ and ${ }_{19} \mathrm{~F}$ nuclei in the Earth's magnetic field.

- Quantification of heteronuclear coupling in the Earth's field, with comparison to that measured at higher fields.

- Quantification of $\mathrm{T}_{1}$ and $\mathrm{T}_{2}$ relaxation times for ${ }_{1} \mathrm{H}$ nuclei in different chemical environments.

- Calculation of $\mathrm{r}_{1}$ and $\mathrm{r}_{2}$ relaxivity values for paramagnetic metal ions.

- Acquisition of one- and two-dimensional MRI images in the Earth's magnetic field.

The experiment is aimed at undergraduate students at an advanced level of study in chemistry, who will already have covered theoretical and practical applications of NMR spectroscopy in their lecture courses. After completion of the session, students should be able to:

- Explain the importance of a homogeneous magnetic field in NMR spectroscopic measurements and its effect on the resulting spectra.

- Understand the limitations of NMR measurements in the Earth's magnetic field.

- Describe spin-lattice and spin-spin relaxation processes, their measurement, and relation to differences in acquired spectra.

- Explain the appearance of the spectra that result from ${ }_{1} \mathrm{H}-19 \mathrm{~F}$ and ${ }_{1} \mathrm{H}-14 \mathrm{~N}$ coupling, with comparison to high field measurements. 
- Describe the relationship between paramagnetic ion concentration and relaxivity.

- Explain how NMR spectroscopy relates to MRI and how weighted imaging may be achieved.

\section{EXPERIMENT OVERVIEW}

Students carry out the experiment individually over six hours, which includes a break of their own

115 planning (e.g., for lunch). Pre-lab questions and Control of Substances Hazardous to Health (COSHH) assessments are set to be completed by students prior to beginning the practical session. These questions ensure students recap the fundamentals of NMR spectroscopic theory, such as the relationship between gyromagnetic ratio, Larmor frequency, and magnetic field strength. The importance of magnetic field homogeneity for NMR spectroscopy is also brought to the students' attention, as it is a key concept in EFNMR spectroscopy. Students then work through the experimental procedure provided, investigating the aspects of NMR spectroscopy discussed below.

\section{NMR Spectroscopy}

Students begin by performing simple acquisitions of the $[1 \mathrm{H}]$ NMR spectra of water, oil, and ethanol in the Earth's field. Running a pulse-and-collect experiment on a water sample yields a free induction

125 decay (FID) and the resulting frequency spectrum. The corresponding field strength can be calculated, reinforcing the relationship between field strength, precession frequency, and gyromagnetic ratio. Attention can also be drawn to features such as the magnitude and full width at half maximum (FWHM) intensity, which depend on the homogeneity of the field across the sample. Performing a spinecho experiment then demonstrates how improving the phase coherence of the net magnetization can

130 result in an increased signal amplitude and narrower peak (lower FWHM). Students are asked to draw out a spin-echo pulse sequence in the pre-lab assessment and should be able to discuss how this improvement in signal occurs. Similar acquisitions for oil and ethanol samples also result in a single signal. For oil, the signal is significantly weaker than for water, introducing the concept of differing relaxation times, whilst for ethanol the single singlet demonstrates that chemical shifts and

135 homonuclear coupling are not observed in the Earth's field. 

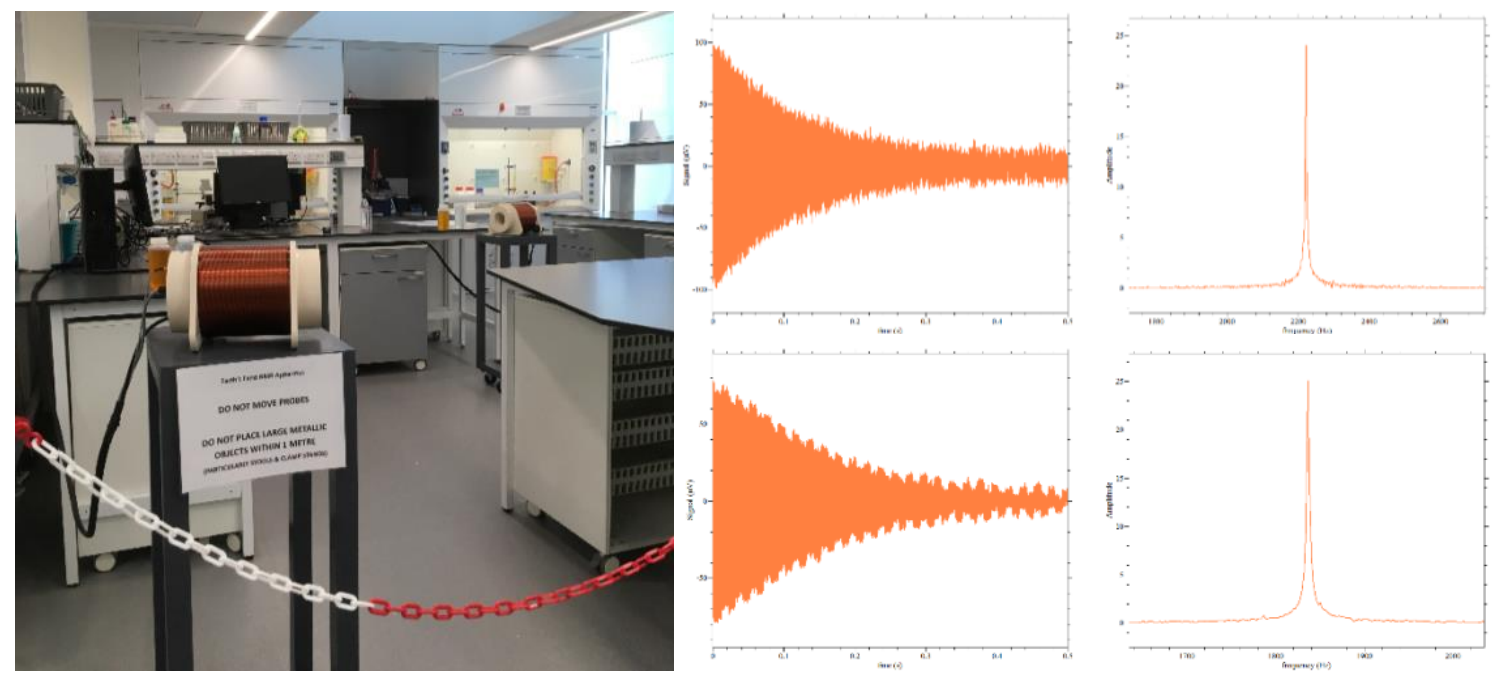

Figure 1. Left: Terranova-MRI probes located within the chemistry teaching labs. Right: ${ }_{1} \mathrm{H}$ NMR signals recorded on both probes simultaneously. Variation in location and shimming results in different observed Larmor frequencies. Data collected by the authors.

Most interestingly from a chemistry perspective, heteronuclear coupling is field independent and so

can be observed by EFNMR spectroscopy. It is also possible to observe multiple nuclei in the same spectral region provided the gyromagnetic ratio for the nuclei are close to that of ${ }_{1} \mathrm{H}(42.58 \mathrm{MHz} \mathrm{T}-1$, 99.985\% abundance). In particular, ${ }_{19} \mathrm{~F}$ EFNMR spectroscopy is possible given the gyromagnetic ratio of $40.05 \mathrm{MHz} \mathrm{T}_{-1}$ and $100 \%$ abundance. Molecules such as 1,4-difluorobenzene can be used to demonstrate that both ${ }_{1} \mathrm{H}$ and ${ }_{19} \mathrm{~F}$ NMR signals can be observed in the same EFNMR spectrum, along with the coupling between them. Coupling of $1 \mathrm{H}$ to nuclei with spin quantum numbers other than one half can also be observed, for example, the coupling to ${ }_{14} \mathrm{~N}(\mathrm{I}=1)$ in $\left[\mathrm{NH}_{4}\right]_{+}$. Here, the ${ }_{14} \mathrm{~N}$ signal cannot be detected as the low gyromagnetic ratio (3.08 $\mathrm{MHz}$-1) gives rise to a Larmor frequency outside of the receiver range of the EFNMR spectrometer.

\section{Relaxivity Measurements}

Investigation of vegetable oil yields NMR spectra that differ from those of water, with lower signal magnitude in a pulse-and-collect experiment, and no signal observed by spin-echo. Students are led to the conclusion that this is due to differences in the relaxation times for protons in each sample. The concept of spin-lattice $\left(\mathrm{T}_{1}\right)$ relaxation is introduced by systematically varying polarization time, $t$, and noting the resulting amplitude. Typically, spin-lattice relaxation is only thought about as the time taken for the net magnetization to return from the $\mathrm{x}-\mathrm{y}$ (transverse) plane after a $90^{\circ}$ pulse. However, more generally it is the time taken for the net magnetization to reach equilibrium after some perturbation. Students can plot the change in signal magnitude as a function of polarization time 
according to Equation 1, where $M_{t}$ is the signal magnitude for a given value of $t$, and $M_{\max }$ is the maximum magnitude observed after infinitely long $t$. From this, a rudimentary value of $\mathrm{T}_{1}$ for water can be calculated. A similar treatment for the vegetable oil data is not possible as the polarization duration is not lowered sufficiently to observe a reduced magnitude.

$$
\begin{gathered}
M_{t}=M_{\max }\left(1-e^{-t / T_{1}}\right) \\
M_{\text {echo }}=M_{0} e^{-t_{\text {echo }} / T_{2}}
\end{gathered}
$$

Spin-spin $\left(\mathrm{T}_{2}\right)$ relaxation is initially introduced through a spin-echo experiment on the vegetable oil sample, which yields no signal. An echo time of $200 \mathrm{~ms}$ is suitable for water $\left(\mathrm{T}_{2}=1700 \mathrm{~ms}\right)$ but longer than the $\mathrm{T}_{2}$ time for oil (100 ms), resulting in a loss of coherence that cannot be recovered by the echo experiment. Measurement of $\mathrm{T}_{2}$ can be achieved be performing a Carr-Purcell-Meiboom-Gill pulse sequence, in which a spin-echo sequence is followed by multiple further $180^{\circ}$ pulses. The intensity of the resulting echoes decreases over time as the signal is gradually lost through randomization of the spin directions via spin-spin energy transfer. Equation 2 defines the exponential decay that links the magnitude of the first echo signal, Mo, the magnitude of subsequent echoes, Mecho, and the echo time, techo.

Next, students explore the effect of paramagnetic ions on the relaxation times of water. $\mathrm{Cu}_{2+}\left(\mathrm{d}_{9}\right)$ and $\mathrm{Mn}_{2+}\left(\mathrm{d}_{5}\right)$ species can be used to demonstrate this effect and the resulting changes to relaxation 175 times measured using pre-programmed experiments as part of the Prospa software (V3.24, Magritek). The degree to which a paramagnetic ion affects the relaxation rates of water is defined as their relaxivity. Both $\mathrm{T}_{1}$ and $\mathrm{T}_{2}$ are measured for a range of concentrations and plotted according to the relationship defined in Equation 3 to determine the relaxivity, $r_{i}$, where $i=1$ or 2 .

$$
\frac{1}{T_{i}}=r_{i}\left[M^{2+}\right]+\frac{1}{T_{i}(0)}
$$

\section{Magnetic Resonance Imaging}

Finally, students carry out MRI in the Earth's field using the gradient coils in the probe.

Application of a magnetic field gradient across the sample results in a range of Larmor frequencies, the exact value of which depends on the position of the proton along the gradient direction. This may be 
185 carried out in three dimensions to obtain images from the NMR signal. Tubes of water, also known as phantoms, are used as the model system. Students record one- and two-dimensional images of a phantom containing two tubes of water to look at the relationship between frequency and position. Subsequent replacement of water for a dilute $\mathrm{CuSO}_{4}$ solution in one of the tubes then allows students to explore the concept of weighted imaging in MRI. Careful manipulation of the polarization duration

190 and echo time parameters can result in selective reduction of the signal for either the standard water or doped-water tube. Students are tasked to think back to the earlier exercises to decide on how the $\mathrm{T}_{1}$ and $\mathrm{T}_{2}$ relaxation times of the two tubes will affect the images they collect.

\section{HAZARDS}

1,4-Difluorobenzene is highly flammable and harmful by inhalation. $\mathrm{CDCl}_{3}$ is irritating to eyes and 195 skin, carcinogenic, and toxic if inhaled. $\mathrm{CuSO}_{4}$ solution may be harmful if swallowed and can cause serious eye irritation. $\mathrm{MnCl}_{2}$ solution is toxic if swallowed and can cause eye damage. $\mathrm{NH}_{4} \mathrm{NO}_{3}$ solution in $\mathrm{HCl}$ can cause serious irritation to eyes and skin. Students should wear gloves along with standard laboratory attire when preparing samples for NMR spectroscopic analysis. A DC current of up to 6 A is pulsed through the outermost polarising coil of this instrument and can heat up if used for extended

200 periods of time. Under normal operating conditions, the surface temperature of the coil should not exceed 40 oC. If excessive heating is observed, the equipment should be turned off and allowed to cool to room temperature.

\section{RESULTS AND DISCUSSION}

The success of EFNMR spectroscopy experiments in the undergraduate chemistry laboratory is

205 dependent on a variety of factors, notably, establishing a reliable set up of the probes and suitable shimming to ensure a homogeneous field. Detailed instructions provided by Magritek were followed for the initial set up although these assume a starting field of high homogeneity. The laboratory, full of instrumentation including fume cupboards, resulted in somewhat inhomogeneous environments for the instruments. Instructions have been put together for set up in such instances, including a list of 210 troubleshooting suggestions to reduce set up time for future implementations of the apparatus. Development of the experiment required ensuring two probes could be run simultaneously without interference, and that both probes gave good quality data in all aspects of the experiment. 
Two $[1 \mathrm{H}]$ EFNMR spectra of water, acquired simultaneously using two probes are shown in Figure 1. Once established, these probes required minimal upkeep provided the immediate magnetic

215 environment remained constant. Small adjustments of the probe shim values were required on only three occasions throughout the eight week term. These adjustments could be carried out within one hour, prior to students starting the experiment.

The NMR spectrum of 1,4-difluorobenzene demonstrated both ${ }_{1} \mathrm{H}$ and ${ }_{19} \mathrm{~F}$ NMR signals, as well as the coupling $(J=6 \mathrm{~Hz})$ between the nuclei (Figures 2a and b). Comparison to spectra recorded at 220 higher fields highlighted the field strength independence of this coupling (Figures $2 \mathrm{c}$ and d). Here, students used $60 \mathrm{MHz}$ benchtop NMR spectrometers for convenience, although the same results could be obtained on a $400 \mathrm{MHz}$ spectrometer. Students also observed good agreement between the results obtained here and spectra reported in the literature.12,16

Figure 2e shows the NMR spectrum recorded for $\left[\mathrm{NH}_{4+}\right]$ in the Earth's field, which contains a triplet 225 corresponding to ${ }_{1} \mathrm{H}-14 \mathrm{~N}$ coupling $(J=53 \mathrm{~Hz}$, Figure $2 \mathrm{e})$. The set up in an undergraduate laboratory with significant background noise prevents complete resolution of the coupling that is observed in the simulated EFNMR spectrum of $\left[\mathrm{NH}_{4}\right]_{+}$at $52.8 \mu \mathrm{T}$ (Figure 2f). Density matrix simulations of the [NH4+] NMR spectrum in the Earth's magnetic field were carried out in Python based on methods described elsewhere.17 A field strength of $52.8 \mu \mathrm{T}$ and a ${ }_{1} \mathrm{H}-14 \mathrm{~N}$ coupling constant of $53 \mathrm{~Hz}$ were used to allow 230 direct comparison to the experimental spectra. Previous work has shown that this splitting occurs because the strong coupling to $14 \mathrm{~N}$ removes the degeneracy of the equivalent ${ }_{1} \mathrm{H}$ nuclei.11 This goes beyond the scope of the undergraduate experiment and it can be seen as beneficial that the fine structure is not observed by the students. Figure $2 \mathrm{~g}$ shows a benchtop NMR spectrum of $\left[\mathrm{NH}_{4}\right]_{+}$ measured in water using a $1 \mathrm{H}$ lock on the spectrometer. Students are asked to consider the problems 235 associated with acquiring a spectrum of $\left[\mathrm{NH}_{4}\right]+$ in $\mathrm{D}_{2} \mathrm{O}$ to highlight some of the limitations that exist in NMR spectroscopy. 

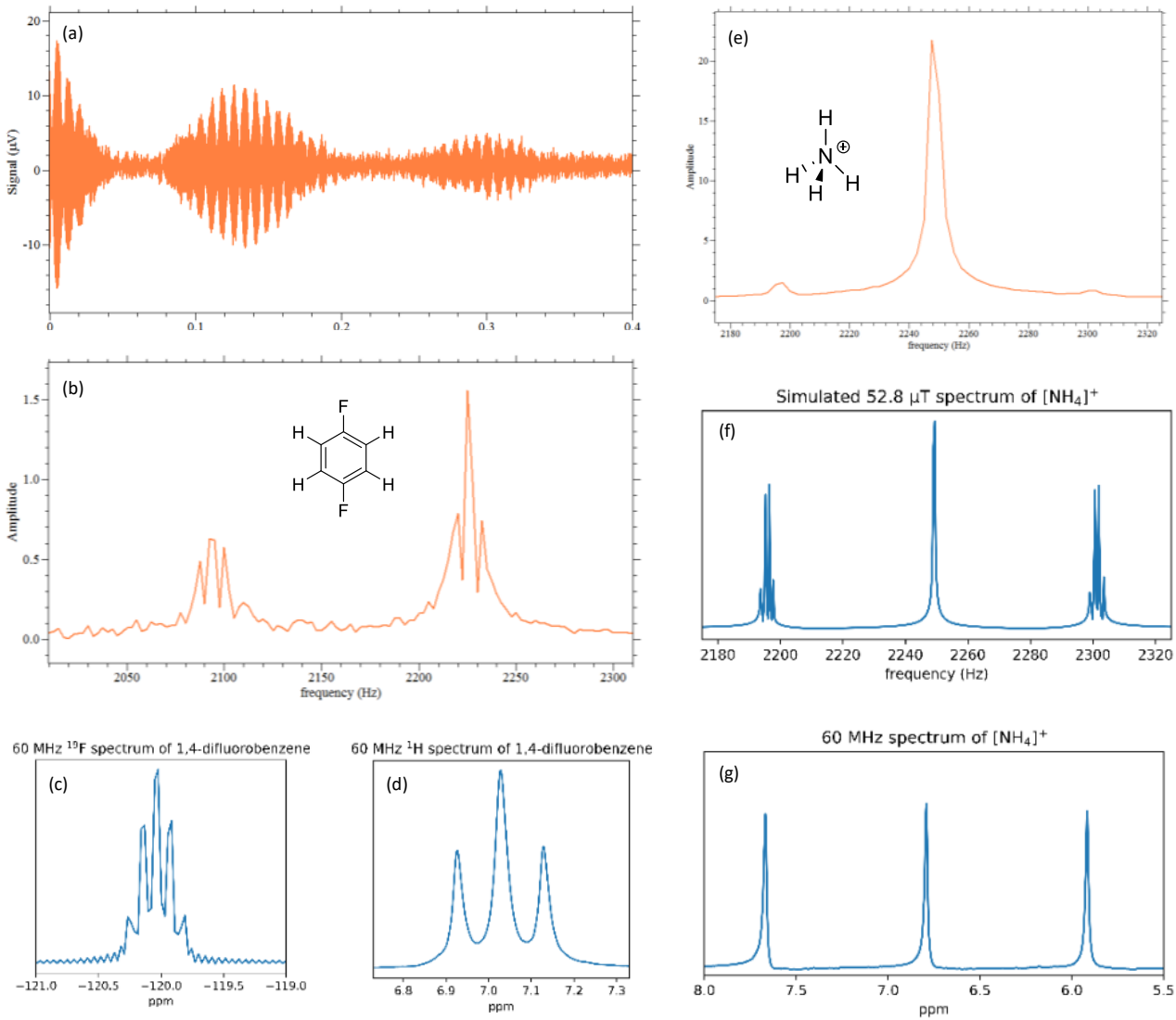

Figure 2. a) FID and b) NMR spectra for 1,4-difluorobenzene, recorded by students in the Earth's field. The corresponding $\mathrm{c}$ ) ${ }_{19} \mathrm{~F}$ and $(\mathrm{d}){ }_{1} \mathrm{H}$ spectra were also recorded using an NMReady-60 Pro benchtop spectrometer $(60 \mathrm{MHz})$. e) NMR spectrum for $\mathrm{NH}_{4+}\left(8 \mathrm{M} \mathrm{NH}_{4} \mathrm{NO}_{3}\right.$ in $\left.2 \mathrm{M} \mathrm{HCl}\right)$ recorded in the Earth's field, and f) simulated NMR spectrum using density matrix calculations, carried out by the authors.11,17 The splitting of the outer two peaks predicted by simulation are not seen in the EFNMR spectrum due to insufficient spectral resolution. g) At $60 \mathrm{MHz}$, a triplet with a coupling constant of $53 \mathrm{~Hz}$ is observed.

Aggregated relaxivity values for $\mathrm{Cu}_{2+}$ and $\mathrm{Mn}_{2+}$, as measured by students, are given in Figure 3, which demonstrate the linear relationship between concentration and the relaxation rate (inverse relaxation time) described by Equation 3. At high concentrations it can become difficult to measure accurate relaxation times as they can be very short, below around $200 \mathrm{~ms}$, resulting in the large deviations observed. Students needed to choose sufficiently short polarization durations or echo times in order to obtain the best fit for the data. Whilst students obtained a range of relaxivity values, the difference between $\mathrm{Cu}_{2+}$ and $\mathrm{Mn}_{2+}$ remained clear, and their relative efficacy as contrast agents could be discussed as part of the post-lab questions. 

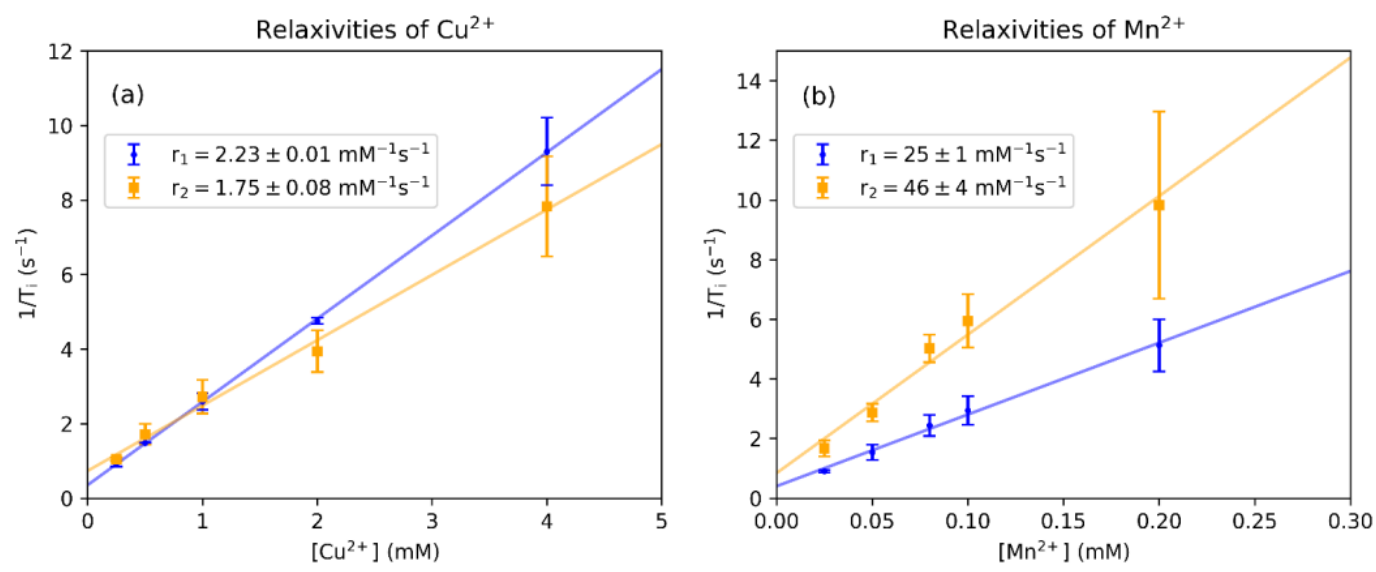

Figure 3. $T_{1}$ and $T_{2}$ relaxation rates measured by the students plotted as a function of a) $\mathrm{Cu}_{2+}$ and b) $\mathrm{Mn}_{2+}$ concentration. Relaxivity values ( $r_{1}$ and $r_{2}$ ) can be calculated from the gradient of the least squares fit and are reported here along with the error in the gradient $(n=14)$. The intercept corresponds to the relaxation rate of pure water and can be verified experimentally.

For the imaging section of the practical session, both one- and two-dimensional NMR images were obtained by students, as shown in Figures $4 \mathrm{~b}$ and c. Students should be able to explain that the abscissa in Figure $4 \mathrm{~b}$ is in fact a spatial axis, related to the gradient applied in the chosen image direction. The two signals arise from the two tubes of water, imaged through the $z$-axis as indicated in Figure 4a. Careful selection of the two-dimensional MRI parameters allows acquisition of suitable images within six minutes. Figure $4 \mathrm{c}$ shows two regions of high intensity that correspond to the twotube phantom, as viewed through the yz plane. Three-dimensional images were not required here due to the prohibitively long acquisition time, which can be upwards of around three hours for a low resolution image volume.

Weighted MRI imaging using water and a dilute $\mathrm{CuSO}_{4}$ solution allowed for a selective reduction in signal intensity, as seen in Figure 5. Based on the relaxivity measurements performed previously, students chose appropriate polarization duration or echo times for both $\mathrm{T}_{1}$ - and $\mathrm{T}_{2}$-weighted imaging. Figure 5 a shows the former case, for which the polarization duration was decreased such that the signal from the tap water (longer $\mathrm{T}_{1}$ ) was attenuated, whilst that from the $\mathrm{Cu}_{2+-}$-doped water was not attenuated (shorter $\mathrm{T}_{2}$ ). It is important to keep the echo time short otherwise the signal from the $\mathrm{Cu}_{2+-}$ doped tube decreased significantly. A long echo time and long polarization time was used for $\mathrm{T}_{2}-$ weighted imaging, in which the signal from the $\mathrm{Cu}_{2+-}$ doped tube is lost through randomization of the nuclear spins by spin-spin relaxation (Figure 5b). 

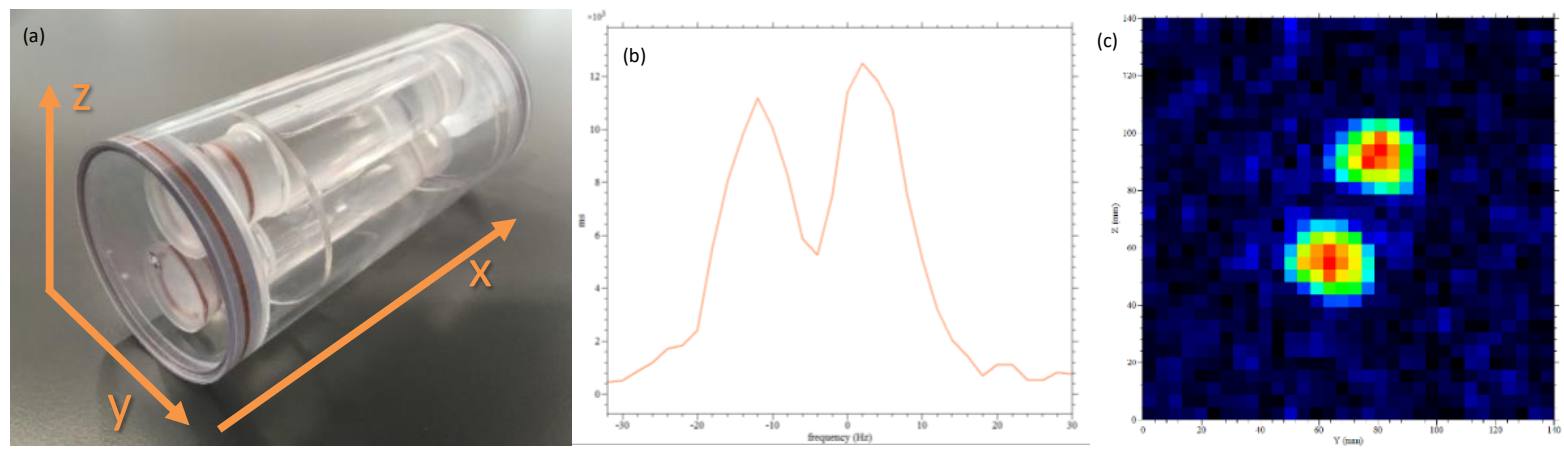

Figure 4. a) The two-tube phantom used for imaging, with the $x, y$, and $z$ imaging axes labelled. b) One- and c) two-dimensional images of a two-tube phantom, in the $z$ and $y z$ directions respectively, recorded using the gradient coils of the Terranova-MRI probe.

(a)

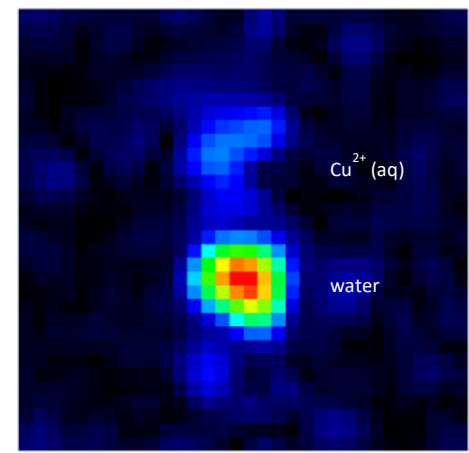

(b)

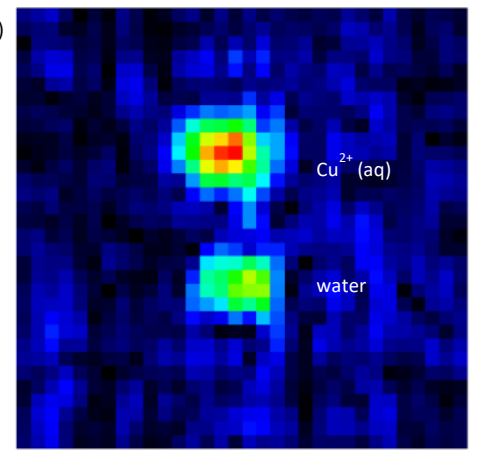

Figure 5. Examples of a) $\mathrm{T}_{1}$ - and b) $\mathrm{T}_{2}$-weighted images of two tubes with $\mathrm{CuSO}_{4}$ solution (top tube) or tap water (bottom tube) acquired by students. Shortened polarization time selectively reduces the tap water signal, whilst a long echo time selectively reduces the $\mathrm{Cu}_{2+}-\mathrm{doped}$ water signal.

The experiment was introduced as an optional third year practical session, running for eight weeks during the spring term. Two students could carry out the experiment simultaneously on any given day, for a potential throughput of 10 students per week. In total, 14 students carried out the practical session over the term, often with two students successfully carrying out the experiments simultaneously. All students were able to complete the experiments within six hours of laboratory time. Table 1 shows the spread of marks awarded to students, with $71 \%$ of students achieving above $70 \%$ (a first class grade) in the post-laboratory assessment. These marks indicated the understanding of the students after completing the practical session was in line with that set out in the learning outcomes. It has been reported that interactive NMR spectroscopy teaching, supplementary to a traditional lecture series, can increase students' theoretical understanding, and in doing so, increase their NMR spectroscopy problem-solving skills.18 The work focused on chemical shift values and multiplicity, although a similar constructivist learning approach likely applies here to relaxation times and pulse sequences. A hands-on approach to NMR spectroscopy is crucial for a thorough 
understanding of the core concepts that enable students to interpret more complicated spectra later in their course or chemical career.

\begin{tabular}{|c|c|c|c|c|}
\hline $\begin{array}{c}\text { Score } \\
(\%)\end{array}$ & Pre-Lab & In-Lab & Post-Lab & Overall \\
\hline $0-50$ & 0 & 0 & 0 & 0 \\
\hline $51-60$ & 0 & 0 & 2 & 0 \\
\hline $61-70$ & 3 & 4 & 2 & 3 \\
\hline $71-80$ & 7 & 8 & 5 & 9 \\
\hline $81+$ & 4 & 2 & 5 & 2 \\
\hline
\end{tabular}

Student feedback to this new practical session was encouraging, with students noting the experiments helped them understand various aspects of NMR spectroscopy more clearly. Students unanimously reported the MRI exercise as the highlight of the session, both because of the visual impact and the clear relation to simple NMR spectrum acquisition. As part of the post-lab questioning, the relaxivity measurements and weighted imaging can be linked to the development of new contrast agents for NMR, which is an active area of current research.19 Specifically, the use of Gd3+ chelates in clinical MRI can be discussed, which the majority of students identify as an effective paramagnetic contrast agent as a result of its seven unpaired electrons. 20

\section{CONCLUSIONS}

305 NMR in the Earth's field offers a unique opportunity to link theory to practice for undergraduate students. A comprehensive practical session has been reported that covers a number of key aspects of NMR spectroscopy that relate to chemistry and chemical research. Multiple probes have been established within an undergraduate chemistry laboratory and can be used to perform both spectroscopy and imaging experiments. Students demonstrated achievement of the learning outcomes and their understanding of NMR strengthened as a result of the experiments.

\section{ASSOCIATED CONTENT}

Supporting Information

The Supporting Information is available on the ACS Publications website at DOI:

\subsection{1/acs.jchemed.9b00359}


Demonstrator Notes (DOCX)

\section{AUTHOR INFORMATION}

Corresponding Authors

*E-mail: patrick.bergstrommann@chem.ox.ac.uk; malcolm.stewart@chem.ox.ac.uk

\section{ACKNOWLEDGMENTS}

We would like to thank laboratory technicians Harry Lingard-Bright and Thomas Throup for testing the experiment and their constructive feedback.

\section{REFERENCES}

1. Riegel, S. D.; Leskowitz, G. M. Benchtop NMR Spectrometers in Academic Teaching. TRAC-

2. Blümich, B.; Singh, K. Desktop NMR and Its Applications From Materials Science To Organic Chemistry. Angew. Chem., Int. Ed. 2018, 57 (24), 6996-7010.

3. Zivkovic, A.; Bandolik, J. J.; Skerhut, A. J.; Coesfeld, C.; Zivkovic, N.; Raos, M.; Stark, H. Introducing Students to NMR Methods Using Low-Field ${ }_{1} \mathrm{H}$ NMR Spectroscopy to Determine the Structure and the Identity of Natural Amino Acids. J. Chem. Educ. 2017, 94 (1), 115-120.

4. Isaac-Lam, M. F. Analysis of Bromination of Ethylbenzene Using a $45 \mathrm{MHz}$ NMR Spectrometer: An Undergraduate Organic Chemistry Laboratory Experiment. J. Chem. Educ. 2014, 91 (8), 1264-1266.

5. Kent, J. E.; Bell, N. G. A. Molecular Properties of Caffeine Explored by NMR: A Benchtop NMR Experiment for Undergraduate Physical-Chemistry Laboratories. J. Chem. Educ. 2019, 96 (4), 786-791.

6. Yearty, K. L.; Sharp, J. T.; Meehan, E. K.; Wallace, D. R.; Jackson, D. M.; Morrison, R. W. Implementation of PicoSpin Benchtop NMR Instruments into Organic Chemistry Teaching Laboratories through Spectral Analysis of Fischer Esterification Products. J. Chem. Educ. 2017, 94 (7), 932-935.

7. Bonjour, J. L.; Hass, A. L.; Pollock, D. W.; Huebner, A.; Frost, J. A. Bringing NMR and IR Spectroscopy to High Schools. J. Chem. Educ. 2017, 94 (1), 38-43.

8. Packard, M.; Varian, R. Free Induction in the Earth's Magnetic Field. In American Physical Society, Minutes of the Stanford Meeting December 28, 29 and 30, 1953; Kaplan, J., Ed.; Phys. Rev. 1954, 93 (4), Abstr. A7, p 941.

9. Waters, G. S. A Measurement of the Earth's Magnetic Field by Nuclear Induction. Nature 1955, 176 (4484), 691.

10. Mohorič, A.; Stepišnik, J. NMR in the Earth's Magnetic Field. Prog. Nucl. Magn. Reson. Spectrosc. 2009, 54 (3-4), 166-182.

350 11. Halse, M. E.; Callaghan, P. T.; Feland, B. C.; Wasylishen, R. E. Quantitative Analysis of Earth's Field NMR Spectra of Strongly-Coupled Heteronuclear Systems. J. Magn. Reson. 2009, 200 (1), 
88-94.

12. Robinson, J. N.; Coy, A.; Dykstra, R.; Eccles, C. D.; Hunter, M. W.; Callaghan, P. T. TwoDimensional NMR Spectroscopy in Earth's Magnetic Field. J. Magn. Reson. 2006, 182 (2), 343347.

13. Benli, K. P.; Dillmann, B.; Louelh, R.; Poirier-Quinot, M.; Darrasse, L. Illustrating the Quantum Approach with an Earth Magnetic Field MRI. Eur. J. Phys. 2015, 36 (3), Article 035032.

14. MRI: Earth's Field NMR and Magnetic Resonance Imaging https://www.physics.utoronto.ca/ phy326/mri/ (accessed Aug 2019).

360 15. Erhart, S. E.; McCarrick, R. M.; Lorigan, G. A.; Yezierski, E. J. Citrus Quality Control: An NMR/MRI Problem-Based Experiment. J. Chem. Educ. 2016, 93 (2), 335-339.

16. Paterson, W. G.; Wells, E. J. NMR Spectrum of Para-Difluorobenzene. J. Mol. Spectrosc. 1964, 14 (1-4), 101-111.

17. Halse, M. E. Multi-Acquisition and Multi-Dimensional Earth's Field Nuclear Magnetic Resonance Spectroscopy. Ph.D. Dissertation, Victoria University of Wellington, 2009.

18. Supasorn, S.; Vibuljan, S. Use of an Interactive NMR Spectroscopy Course to Enhance the NMR Understanding of University Students. J. Online Learn. Teach. 2009, 5 (3), 458-468.

19. Zheng, X. Y.; Pellico, J.; Khrapitchev, A. A.; Sibson, N. R.; Davis, J. J. Dy-DOTA Integrated Mesoporous Silica Nanoparticles as Promising Ultrahigh Field Magnetic Resonance Imaging Contrast Agents. Nanoscale 2018, 10 (45), 21041-21045.

20. Clough, T. J.; Jiang, L.; Wong, K. L.; Long, N. J. Ligand Design Strategies to Increase Stability of Gadolinium-Based Magnetic Resonance Imaging Contrast Agents. Nat. Commun. 2019, 10 (1), $1-14$. 